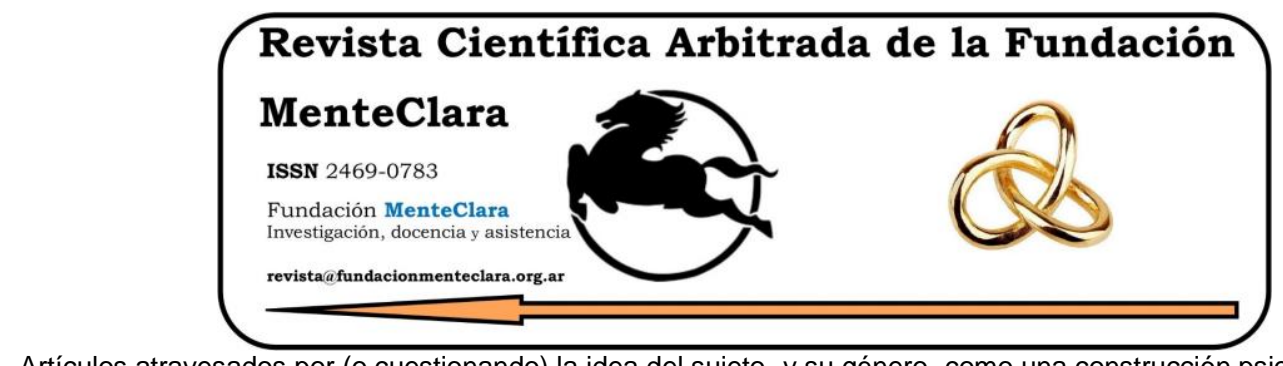

Artículos atravesados por (o cuestionando) la idea del sujeto -y su género- como una construcción psicobiológica de la cultura. Articles driven by (or questioning) the idea of the subject -and their gender- as a cultural psychobiological construction. Vol. 5 (2020), enero-diciembre ISSN 2469-0783

https://datahub.io/dataset/2020-5-e200

\title{
INFLUENCIA DE LA MOTIVACIÓN EN LA ACTIVIDAD FISICA, EL DEPORTE Y LA SALUD
}

\author{
INFLUENCE OF MOTIVATION ON PHYSICAL ACTIVITY, SPORTS, AND HEALTH
}

Edgardo Pereyra edgardo.pereyra@uflouniversidad.edu.ar

Facultad de Psicología y Ciencias Sociales, Universidad de Flores, Argentina

Cómo citar este artículo / Citation: Pereyra, E. (2020). Influencia de la motivación en la actividad fisica, el deporte y la salud. Revista Científica Arbitrada de la Fundación MenteClara, Vol.5 (200). DOI: https://doi.org/10.32351/rca.v5.200

Copyright: (C) 2020 RCAFMC. Este artículo de acceso abierto es distribuido bajo los términos de la licencia Creative Commons Attribution 4.0 International License (CC BY 4.0). Recibido: 29/11/2020. Aceptado: 07/12/2020 Publicación online: 10/12/2020

Conflicto de intereses: Ninguno que declarar.

\section{Resumen}

La motivación como aspecto psicológico tiene una gran interacción con el desarrollo del ser humano. Al ser un estado y no un rasgo la misma puede variar en distintos momentos y situaciones, siendo de característica fluctuante y dinámica. Además, la misma puede ser regulada de manera interna y/o externa a través de diversos condicionantes que determinan la capacidad de fortalecerla o disminuirla según sea el propósito. La finalidad del siguiente trabajo es exponer análisis y resultados de como la variable motivación influye en el desarrollo actitudinal y en la modificación de hábitos de las personas hacia el direccionamiento, mantenimiento y consagración de logros u objetivos en ámbitos de actividad física, deporte y salud en diversas poblaciones.

\begin{abstract}
Motivation as a psychological aspect has a great interaction with the development of the human being. Being a state and not a trait, it can vary at different times and situations, being fluctuating and dynamic. In addition, it can be regulated internally and / or externally through various conditions that determine the ability to
\end{abstract}


strengthen or reduce it depending on the purpose. The purpose of the following work is to present the analysis and results of how the motivation variable influences attitudinal development and the modification of people's habits towards the direction, maintenance and consecration of achievements or objectives in areas of physical activity, sport and health in various populations.

Palabras Claves: Motivación; Actividad Física; Deporte; Educación; Hábitos Saludables Keywords: Motivation; Physical Activity; Sport; Education; Healthy Habits 


\section{Introducción}

Para desarrollar el siguiente trabajo se realizó una integración y análisis de distintos estudios científicos donde la variable utilizada para la búsqueda fue la motivación. Cabe señalar que naturalmente la motivación es un factor que atraviesa, en mayor o menor medida, al ser humano a lo largo de su existencia, sin diferenciar rangos etarios, culturas, razas, ni condiciones físicas. Por otra parte, siendo la motivación un concepto de amplio alcance, el presente trabajo va a estar meramente centrado en los conceptos de motivación extrínseca y motivación intrínseca o autodeterminada. A tal fin la variable será analizada dentro de espacios y poblaciones determinadas por los estudios recabados.

\section{Motivación}

Desde el punto de vista etimológico, la palabra Motivación está compuesta por el latín Motivus -movimiento- y el sufijo ción -acción y efecto-.

Según la Real Academia Española la motivación es un conjunto de factores internos o externos que determinan en parte las acciones de una persona.

En una breve definición Woolfolk (2010) señala que la motivación es un estado interno que incita, dirige y mantiene la conducta.

Ya en una definición más extensa, González Serra (2008) menciona que la motivación es el conjunto concatenado de procesos psíquicos que al contener el papel activo y relativamente autónomo y creador de la personalidad, y en su constante transformación y determinación reciprocas con la actividad externa, sus objetos y estímulos, van dirigidos 
a la satisfacción de las necesidades del ser humano y, como consecuencia, regulan la dirección y la intensidad del comportamiento.

Cabe destacar que la motivación en sí, no puede ser observada, sino que lo que se observa el comportamiento de un ser motivado y de dicho comportamiento se infiere la existencia de la motivación.

\section{Motivación extrinseca}

Según Ryan y Deci (2000) cuando existe algún tipo de recompensa o interés ajeno a la propia acción del individuo y esto motiva a la realización de una actividad con el fin de lograr un objetivo, se la denomina motivación extrínseca. Esta motivación está más destinada a conseguir la recompensa o el interés en sí, que en buscar la propia satisfacción o desarrollo del individuo.

\section{Motivación intrínseca o autodeterminada}

Para Ambrose y Kulik (1999) la motivación intrínseca es la verdadera motivación y por lo tanto la que debiera buscarse manifestar en las personas para lograr un cambio o progreso verdadero en su comportamiento. Estar motivado intrínsecamente es asumir un problema como reto personal, es enfrentar una situación sin esperanza o anhelo de que haya una recompensa externa. Esta motivación está relacionada con aspectos más profundos de crecimiento y autoconocimiento.

\section{Algunas teorias referentes a la motivación}

De acuerdo a diferentes teorias relacionadas a la motivación, vamos a hacer referencia a las que de alguna manera serán articuladas en los 
análisis de estudios recabados que se expondrán posteriormente en este trabajo.

\section{Teoria de la Pirámide de las Necesidades}

Esta teoria propuesta por Maslow (1943) se basa en que cada ser humano se esfuerza para satisfacer necesidades escalonadas. Los distintos escalones se representan en forma de pirámide y son los siguientes:

Necesidades Fisiológicas: Son las necesidades más básicas para mantenerse vivo, respirar, comer, beber, dormir.

Necesidades de Seguridad: Se vinculan con el sentirse seguro, sin peligro.

Necesidades de Pertenencia -sociales-: Necesidades de relaciones humanas, ser integrante de un grupo, recibir afecto de familiares, amigos y personas del sexo opuesto.

Necesidades de Estima: Necesidad de sentirse digno, respetado, valorado.

Necesidades de Autorrealización: Necesidad de crecimiento personal, desarrollo profesional. Podemos incluir la autonomia, la independencia y el autocontrol.

A medida que las necesidades básicas se satisfacen van surgiendo las de orden superior. Es importante remarcar que para pasar a una necesidad superior previamente debe ser satisfecha la necesidad inferior. 


\section{Teoria de la Motivación - Higiene}

En esta teoria Herzberg (1954), apunta a que el rendimiento de en las personas varia en función del nivel de satisfacción.

A través de diversas investigaciones Herzberg llego a la conclusión de que existen dos grupos de factores que pueden crear satisfacción o insatisfacción a la hora de realizar una tarea, estos son los de higiene y los de motivación.

A los factores motivacionales los denomino intrínsecos y a los de higiene, extrínsecos.

Factores motivacionales -intrínsecos-: Reconocimiento, responsabilidad, logro y desarrollo personal, la tarea en sí.

Factores de higiene -extrínsecos-: Clima en el que se realiza la tarea, administración, relación con los líderes, status, recompensa, seguridad en el puesto ocupado.

\section{Teoria de las necesidades de McClelland}

McClelland (1961) basa la teoria en tres necesidades adquiridas:

Necesidades de realización: Interés por desarrollarse, destacarse aceptando responsabilidades personales. Buscan enfrentarse con problemas y afrontar el triunfo o el fracaso.

Necesidades de poder: EL rasgo más marcado es el de tener influencia y control sobre los demás. Prefieren la lucha y la competencia más que los resultados.

Necesidades de filiación: Su característica es ser solicitados y aceptado por los demás. Persiguen la amistad y la cooperación en lugar de la lucha. 


\section{Teoria de la motivación autodeterminada}

Esta teoría también conocida como SDT (Ryan \& Deci, 2000) se basa en la motivación humana inherente al crecimiento y las tendencias innatas y necesidades psicológicas de las personas. Se centra principalmente en el grado en que el comportamiento del individuo es automotivado y autodeterminado direccionado a realizar una actividad porque es interesante y satisfactoria en sí misma. Cabe destacar que esta motivación es contrapuesta a la que se produce cuando el fin es un interés externo, ya que en la misma la motivación se estaría produciendo por la influencia externa.

Los autores también sostienen que hay tres necesidades psicológicas que están involucradas con la autodeterminación y son las que motivan a uno mismo a iniciar el comportamiento e incentivar los nutrientes que son claves para la salud psicológica y el bienestar del ser humano. Dichas necesidades son universales, innatas y psicológicas y son la necesidad de competencia, la autonomía y relación psicológica.

\section{Materiales y análisis}

Teniendo en cuenta los estudios obtenidos se va a realizar un recorrido acercando una articulación con las teorias anteriormente mencionadas, evidenciando así, los resultados expuestos en dichos artículos con sus respectivos métodos e instrumentos de medición.

Para empezar, vamos a mencionar el relevante artículo de (López Walle, et al., 2011) en el cual se busca determinar las variables que influyen en el clima motivacional percibido, la motivación auto determinada y la autoestima en jóvenes deportistas mexicanos. Para la investigación la muestra estuvo compuesta por 1052 deportistas mexicanos participantes en la olimpiada Nacional Mexicana Infantil y 
juvenil se avaluó utilizando como instrumento de medición tres cuestionarios diferentes específicos a cada ítem mencionado.

Los resultados arrojaron por una parte que la motivación autodeterminada es mayor cuando se percibe que los entrenadores generan un clima de implicación en la tarea. Mientras que contrariamente, cuando los deportistas percibian un clima de implicación en el ego, manifestaron una menor motivación autodeterminada. Por otra parte, los resultados también demuestran que los deportistas presentan patrones motivacionales más adaptativos cuando perciben valoración de esfuerzo por parte de sus entrenadores y se sienten tenidos en cuenta como pieza fundamental en la conformación del equipo.

Para finalizar este apartado, podemos también comentar que la autoestima en los deportistas puede ser desarrollada por parte del entrenador a través de la motivación, y que una de las vías para lograrlo, es que este último genere un clima motivacional de implicación en la tarea en el que el deportista pueda ser más autónomo o autodeterminado (López Walle, et al., 2011).

Continuando en la línea de la práctica deportiva tomaremos el trabajo de (Fernández \& Soidán 2010) donde dentro del mismo se investigó cuáles son las motivaciones que llevan a estudiantes universitarios de Galicia a realizar actividad fisica y también el grado de conocimiento que poseen sobre la oferta deportiva en su universidad. Para dicho estudio se tomó como muestra a 716 universitarios gallegos y como instrumento de medición, un cuestionario sobre hábitos de práctica físico-deportiva y otro para comprobar si el alumnado conocía la oferta de los servicios deportivos universitarios. Los resultados demuestran que los universitarios gallegos practicantes de actividad físico-deportiva se sienten motivados a realizar la práctica principalmente por la variable 
lúdica $44 \%$, seguido por la variable relacionada a la salud $35 \%$, y un grupo minoritario por las variables relacionadas a la competencia $8 \%$, lo social 5\%, la estética $5 \%$ y otras cuestiones 1\%. Es importante remarcar que dentro de quienes no realizan actividad físico-deportiva el $71 \%$ acusa no realizarlo por falta de tiempo. En cuanto a la percepción de información de la oferta deportiva en las universidades el 51\% de los universitarios practicantes afirma manejar información suficiente sobre la oferta de los servicios deportivos de su Universidad. (Fernández \& Soidán 2010).

Acercando la propuesta a estudios de motivación, en este caso en un deporte específico como el maratón, tomaremos el artículo de (Ruiz Juan \& Zarauz Sancho, 2014) donde se buscó determinar la motivación que lleva deportistas españoles corredores de maratón, a realizar dicha competencia. García Verdugo y Landa (2004) sostienen que, para concluir un maratón, se requiere un nivel de consumo de oxígeno y un nivel de resistencia superior al de una persona no entrenada, y que solo se desarrolla tras extensos y rigurosos meses o años de entrenamiento con una alta motivación.

La muestra para este estudio estuvo conformada por 1226 corredores de 3 maratones a través del cuestionario de las Motivations of Marathoners Scales-34. Dicho cuestionario contiene 7 subescalas motivacionales compuestas por 34 items o motivos para correr un maratón, que miden el nivel de motivación para correr por cada factor mencionado. Los resultados obtenidos arrojaron que esta población tiene un alto grado de motivación intrínseca o asociadas a la tarea -significado de la vida-autoestima y orientación a la salud- y en menor medida se observaron resultados asociados a la motivación extrínseca o relacionadas con el ego -reconocimiento- (Ruiz Juan \& Zarauz Sancho 2014). 
En cuanto a la motivación para realizar deporte dentro de la etapa de edad escolar en el artículo de (Medrano \& Mateos, 2018) se busca conocer cuáles son los principales factores motivacionales en estos adolescentes para realizar deporte. Los autores expresan que una de las principales ventajas que presenta el deporte en edad escolar, es el interés inicial que el mismo genera, debido a diversas razones, como ser la actividad fisica y lúdica muy gratificante en estas edades, generándose adherencia y compromiso hacia la práctica físico-deportiva en el tiempo libre, y sumado a esto, logrando interacción que se produce con otros compañeros de la misma edad termina siendo un aspecto muy valioso. Entre los datos relevados se evidencio que la motivación es mayor cuando se realizan deportes colectivos o en conjunto, por sobre los deportes individuales y que es necesario potenciar el deporte en edad escolar ya que estos generan los motivos de práctica más intrinsecos y se ha demostrado que esta motivación se vincula con una menor percepción de conflicto entre la práctica deportiva, el contexto escolar y la amistad. (Medrano \& Mateos, 2018). Para el mencionado estudio se tomó como muestra a 816 personas de entre 12 y 18 años que practican diferentes deportes individuales y colectivos en Castilla - La Mancha.

Continuando con la actividad fisica y mencionando el artículo de (Carron, et al., 2016) se busca conocer que variables motivacionales pertenecientes a la teoría de la autodeterminación eran predictivas en etapas de cambio de ejercicio más activas.

Los autores dentro de la introducción refieren que uno de los temas que más preocupan a los investigadores del comportamiento humano es determinar las variables que generen disminución o desaparición de conductas no saludables, como así también que variables hacen que las personas se involucren en un estilo de vida más activo (Carron, et al., 2016). Se deduce de gran importancia poder encontrar recursos que no 
solo permitan acercar a las personas a realizar actividad fisica, sino que también la misma pueda ser sostenida en el tiempo. Este estudio estuvo enmarcado por las teorias de la autodeterminación, ya mencionada anteriormente y también el modelo transteórico del cambio del ejercicio físico (Prochaska \& DiClemente, 1983) el cual se sugiere que el cambio de comportamiento ocurre en el tiempo a través de las etapas, e incluyen procesos cognitivos y comportamentales, de manera que la motivación será un factor que determine la posibilidad de pasar de un estadio a otro, y que no siempre se progresa en etapa lineal, sino que se puede recaer y repetir.

Para este trabajo se tomó como muestra a 187 estudiantes en clases de educación fisica pertenecientes a tres centros de educativos de educación secundaria obligatoria de la ciudad de Badajoz. Se utilizó como instrumento de medición la Escala de Locus Percibido De Causalidad en Educación Física -PLOC Scale- que mide las diferentes formas de motivación establecida por la teoría de la autodeterminación, a través de 20 items.

Parte de la conclusión arrojo que la autonomía, como necesidad básica, es la principal variable motivacional pronosticadora de la etapa de cambio de ejercicio físico de acción, y la competencia, también como necesidad básica, es la pronosticadora motivacional más fuerte de la etapa de ejercicio de mantenimiento.

Sumado a lo anterior y para finalizar se concluyó que diversos estudios afirman que al verse satisfechas las necesidades psicológicas básicas de autonomía, competencia y relaciones sociales, se produce un aumento de la motivación intrínseca de los participantes, y todo esto favorece una mayor práctica de actividad física y deportiva (Carron, et al., 2016). 
Haciendo una interrelación entre la actividad física y el rendimiento escolar el artículo de (González \& Portolés, 2014) busca revelar cuales son las consecuencias motivacionales que puede generar realizar actividad fisica extra escolar y como también esto impacta en la mejora del rendimiento académico y en las conductas asociadas a la salud. Los autores del artículo dicen que el descenso de los niveles de actividad físico-deportiva, así como el incremento del consumo de sustancias nocivas impactan de manera altamente negativa en los adolescentes, además de tener influencia en circunstancias como el rendimiento y la motivación dentro del ámbito académico.

Para el mencionado estudio se presentó una muestra de 421 adolescentes de ESO y Bachillerato de la ciudad de Cartagena, Murcia, España y como instrumento de predicción se utilizaron cuestionarios sociodemográficos, la Escala de Motivación Educativa -EME-S- y diferentes calificaciones escolares.

La conclusión del trabajo entre otros datos arrojo que se evidencia una clara prevalencia a favor del género masculino en cuanto a la práctica de AFYDE, y que las mayores tasas de AFYDE se producen entre los más jóvenes, y estas disminuyen al incrementarse la edad.

Por otro lado, se concluyó que el ser activo ejerce en los adolescentes un efecto protector y preventivo frente a conductas poco saludables, mientras que ser sedentario es un factor de riesgo para la frecuencia de consumo de sustancias nocivas.

Para finalizar, otro dato relevante que arrojo el estudio es que los individuos que indicaron tener menores consumos de alcohol, tabaco y cannabis, demostraron tener mayor rendimiento académico. Esto confirma que la actividad físico-deportiva está asociada a mayor 
rendimiento académico y a la aversión de conductas nocivas que afectan al mencionado rendimiento académico (González \& Portolés, 2014).

Así como el ejercicio físico es una necesidad en ámbito de salud para todas las poblaciones, tomaremos como ejemplo el relevante trabajo de (Pardo, et al., 2014), en el que se busca determinar cuáles son los motivos de práctica de actividad física en adultos mayores. Este estudio está enmarcado teóricamente por la ya mencionada motivación autodeterminada, aunque también dentro del análisis puede asociarse las teorias de la pirámide de Maslow y la teoría de Motivación - Higiene de Herzberg.

El nivel de motivación más o menos autodeterminado del individuo va a estar afectado en gran medida por la interacción de éste con el contexto social, ya sea líderes, pares y diversos agentes sociales. Un contexto de ejercicio físico en el que se le de valor al comportamiento autónomo de los practicantes, podría generar la satisfacción de las necesidades psicológicas básicas -autonomía, competencia y relación con los demás-. Dichas necesidades son mediadoras entre la relación de los factores ambientales y del grado de motivación experimentada (Pardo, et al., 2014).

La muestra para este trabajo estuvo compuesta por 237 practicantes, 94 hombres y 143 mujeres, con edades comprendidas entre los 65 y 85 años pertenecientes a una población de practicantes de diversos centros deportivos de la región de Murcia. Para medir la valoración del comportamiento autónomo se empleó la dimensión de valoración del comportamiento autónomo de (Conde, et al., 2010). Para medir las necesidades psicológicas básicas en el ejercicio se utilizó la versión en español (Sánchez \& Núñez, 2007) de la Escala de las Necesidades Psicológicas Básicas en el Ejercicio (Vlachopoulos \& Michailidou, 2006). Y para medir la motivación hacia la actividad física 
en el contexto del ejercicio físico se utilizó la BREQ-3 (González-Cutre, et al., 2010).

El estudio demuestra, entre otras cuestiones, que planificar metodologias que fomenten la autonomia de los practicantes -tareas abiertas, fomento de la iniciativa, elección de actividades- mejoraría el logro de consecución de las necesidades posológicas básicas, así como una mayor motivación intrínseca.

En concordancia con estos resultados, la teoría de la autodeterminación afirma que el potenciar la autonomía es un factor esencial para la satisfacción de las necesidades psicológicas. De esta manera, si son satisfechas las necesidades psicológicas básicas que median el contexto social, se podría potenciar la motivación intrínseca, que a su vez presente resultados positivos sobre el bienestar y el desarrollo personal (Pardo, et al., 2014).

Así como en este trabajo de análisis ya se dio lugar a la investigación de la motivación para la actividad fisica en poblaciones universitarias, en deportistas y en distintas etapas evolutivas, y teniendo en cuenta que en los últimos años se ha trasformado de manera positiva la percepción social y la inclusión de las personas con discapacidad a distintos ámbitos sociales y culturales, es menester mencionar el artículo de (Toralba, et al., 2014) en el cual se busca determinar cuál es la motivación en el deporte adaptado.

Para este trabajo se tomó como muestra a 134 deportistas de ambos géneros y diferentes discapacidades que forman parte del deporte federado en España y como instrumento de medición se utilizó el cuestionario Participation Motivation Inventory de Gill, Gross y Huddleston que describe las principales razones que incitan a la actividad deportiva. A través del estudio mencionado se busca examinar 
cual es la motivación que lleva a estas personas a realizar deporte, ya que la motivación hacia el deporte ha sido una de las principales preocupaciones de las comunidades deportivas, queriendo llegar a conocer cuáles son los principales motivos que llevan a las personas a practicar un deporte (Toralba, et al., 2014).

Para la muestra se han elegido a personas ciegas o con discapacidad visual, es decir, con alguna característica que impida una visión normal, a personas con alguna discapacidad intelectual, a personas con alguna discapacidad fisica que puede ser alguna articulación principal en un miembro amputado o con una lesión medular, y a personas con parálisis cerebral.

Entre los resultados que arrojó el estudio, sobresale que las principales motivaciones para realizar deporte en esta población son el hecho de practicar deporte, mejorar el nivel, hacer ejercicio, competir, estar fisicamente bien, divertirse, mantenerse en forma, ganar y mejorar las habilidades. Quedando el extremo opuesto los motivos menos valorados que fueron el satisfacer a los padres, ser importante y popular, sentirse importante y realizarlo por influencia de los entrenadores (Toralba, et al., 2014).

Para finalizar este trabajo de análisis es oportuno mencionar el artículo de (Marcos, et al., 2014) donde se busca evidenciar si la motivación grupal tiene efecto en la eficacia del tratamiento del sobrepeso y obesidad. Teniendo en cuenta que la obesidad es una enfermedad crónica multifactorial de gran impacto socio sanitario y económico que constituye un problema de salud pública, es preciso señalar que deja de ser un problema solamente estético cuando se le adjudica un grado en el que aumenta la morbimortalidad y altera la calidad de vida de quien la padece (Marcos, et al., 2014). La misma está asociada muchas veces no solo a malos hábitos de salud en lo relacionado a alimentación, estrés y 
descanso, sino que también está vinculada a una falta de actividad fisica que bien dosificada podría ser de gran ayuda ante esta enfermedad.

El artículo mencionado apunta a evidenciar si la motivación en grupo por profesionales de la enfermería entrenados por un psicólogo experto modifica los hábitos de conductas saludables en la población mencionada previamente. Para la muestra se eligieron de manera aleatoria dos grupos situados en centros diferentes separados de Abacete y Cuenca, uno de los cuales recibió intervención de motivación en grupo y el otro un seguimiento habitual. En los resultados del trabajo se demostró que luego del primer año de seguimiento, la reducción media de peso basal en el grupo control fue del $1.3 \%$ y en el caso del grupo intervención del 1,8\%, pero luego del segundo año de seguimiento la reducción porcentual media del peso se situó en el 1\% para el grupo control y del 2,5\% en el grupo intervención. Esto demuestra que, de alguna manera, la intervención motivacional en forma grupal tiene un cierto impacto positivo para el tratamiento del sobrepeso y la obesidad.

\section{Conclusiones}

Teniendo en cuenta la revisión realizada en las muestras de los artículos previamente expuestos, podemos aseverar que la motivación es una condición indispensable a la hora de modificar conductas, lograr objetivos y generar hábitos saludables en las poblaciones revisadas. Así mismo podemos mencionar que la motivación extrínseca tiene amplia relevancia y se desarrolla de manera positiva si el contexto social y el clima motivacional del entorno resulta facilitador. Sumado a esto y como se ha evidenciado previamente, los reconocimientos, la autonomía y la libertad para elegir acciones influyen en los estados motivacionales, 
dando lugar, a la manifestación de la motivación intrínseca o autodeterminada.

Para finalizar, es oportuno destacar que la motivación intrínseca o autodeterminada parece tener un papel fundamental en el desarrollo actitudinal positivo hacia la realización de tareas y la modificación y mantenimiento de hábitos y conductas saludables en las poblaciones previamente investigadas. 


\section{Referencias}

Ambrose, M., Kulik, C. (1999). Old friends, new faces: motivation research in the1990s. Journal of Management, 25, 231-292.

Carron M., Muñoz S., Román M., Batista M.\& Castuera, R. (2016). Predicción de la motivación en las etapas de cambio de ejercicio más activos. Retos. Nuevas Tendencias en Educación Física, Deporte y Recreación, 30, 87-91.

Conde C., Sáenz-López P., Carmona J., González-Cutre D., Martínez C. \& Moreno J. (2010). Validación del Cuestionario de Percepción de Soporte de la Autonomía en el Proceso de Entrenamiento (ASCQ) en jóvenes deportistas españoles. Estudios de Psicología, $31(2), 145-157$.

Fernández D. \& Soidán J. (2010). Motivación hacia la práctica físico-deportiva de universitarios gallegos. Revista de Investigación en Educación, 8, 128-138.

García-Verdugo, M., \& Landa, L. M. (2004). Atletismo 4: Mediofondo y Fondo (La preparación del corredor de resistencia). Madrid, España: RFEA.

González-Cutre D., Sicilia A., Fernández A. (2010). Hacia una mayor comprensión de la motivación en el ejercicio físico: medición de la regulación integrada en el contexto español. Psicothema, 22 (4) 841-847.

González J. \& Portolés A. (2014). Actividad fisica extraescolar: relaciones con la motivación educativa, rendimiento académico y conductas asociadas a la salud. Revista Iberoamericana de Psicología del Ejercicio y el Deporte, 9 (1), 51-65.

González Serra, D. (2008). Psicología de la motivación. (p. 52). La Habana, Cuba, Editorial Ciencias Médicas.

Herzberg, F. (1954). Work of the Nature of Man. The World of Publishing Company.

López - Walle, J. Balanguer I., Castillo I. \& Tristán J. (2011). Clima motivacional percibido, motivación autodeterminada y autoestima en jóvenes deportistas mexicanos. Revista de Psicología del Deporte, 20 (1), 209-222.

Medrano E. \& Mateos M. (2018). Evaluación de la motivación en adolescentes que practican deportes en edad escolar. Retos. Nuevas Tendencias en Educación Física, Deporte y Recreación. 33, 27-33.

Maslow A. (1943). A Theory of Human Motivation. Psychological Review 50 (4), 370-393. 
McClelland D. (1961). The achieving society. The Free Press.

Pardo P., Castrillón J., Pedreño N. \& Moreno-Murcia J. (2014). Motivación autodeterminada en adultos mayores practicantes de ejercicio físico. Cuadernos de Psicología del Deporte, 14 (3), 149-156.

Prochaska, J. \& DiClemente, C. (1983). Stages and processes of self-change of smoking: Toward an integrative model of change. Journal of Consulting and Clinical Psychology, 51(3), 390-395. https://doi.org/10.1037/0022-006X.51.3.390

Real Academia Española: Diccionario de la lengua española, 23. ${ }^{a}$ ed., [versión 23.3 en linea]. https://dle.rae.es.

Ryan, R. \& Deci, E. (2000). Self-determination theory and the facilitation of intrinsic motivation, social development, and well-being. American Psychologist, 55, 68-78. https://dx.doi.org/10.1037/0003-066X.55.1.68

Ruiz - Juan F. \& Sancho A. (2014). Análisis de la motivación en corredores de maratón españoles. Revista Latinoamericana de Psicología, 46 (1), 1-11. https://doi.org/10.1016/S0120-0534(14)70001-9

Sánchez, J., \& Núñez, J. (2007). Análisis preliminar de las propiedades psicométricas de la versión española de la Escala de Necesidades Psicológicas Básicas en el Ejercicio Físico. Revista Iberoamericana de Psicología del Ejercicio y el Deporte, 2(2), 83-92.

Tárraga Marcos L, Rosich N., Panisello Royo J., Gálvez Casas A., Serrano Selva J., José Antonio Rodríguez Montes J. \& Tárraga López P. (2014). Eficacia de las estrategias de motivación en el tratamiento del sobrepeso y obesidad. Nutrición hospitalaria, 30 (4), 741-748. http://dx.doi.org/10.3305/nh.2014.30.4.7704

Torralba M., Braz M. \& Rubio M. (2014). La motivación en el deporte adaptado. Psychology, Society, \& Education, 6, 27-40.

Valdés Herrera C. (2020). Qué es la motivación, su importancia y sus principales teorias. Recuperado de https://www.gestiopolis.com/motivacion-concepto-y-teorias-principales /

Vlachopoulos, S., \& Michailidou, S. (2006). Development and initial validation of a measure of autonomy, competence, and relatedness in exersice: The Basic Psychological Needs in Exercise Scale. Measurement in Physical Education and Exercise Science, 10, 179-201. https://doi.org/10.1207/s15327841mpee1003_4

Woolfolk, A. (2010). Psicología Educativa. Pearson Educación de México, S.A. 